\title{
Assessment of prostate cancer with dynamic contrast-enhanced computed tomography using an en bloc approach
}

\author{
Huellner, Martin W ; Pauli, Chantal ; Mattei, Agostino ; Ross, Steffen ; Diebold, Joachim ; Vosbeck, \\ Jürg ; Allgayer, Bernhard ; Strobel, Klaus ; Veit-Haibach, Patrick
}

\begin{abstract}
OBJECTIVES The aim of this study was to assess the performance of dynamic contrastenhanced computed tomography of the prostate in patients with biopsy-proven prostate cancer. MATERIAL AND METHODS A total of 46 male patients (median age, 65 years; range, 49-73 years) with biopsy-proven prostate cancer underwent an en bloc computed tomography perfusion (CTP) scan of the prostate before surgery. The perfusion parameters mean transit time (MTT), blood flow (BF), and blood volume (BV), as well as the microvessel density (MVD) of surgical specimens were determined. Differences in CTP parameters and MVD among postsurgical Gleason score (sGS) and postsurgical combined Gleason grade (sGG) groups were analyzed. Spearman correlation coefficients were determined between CTP parameters and presurgical biopsy-derived Gleason score (bGS), presurgical biopsy-derived combined Gleason grade (bGG), sGS, sGG, MVD, and pathological tumor stage. A linear regression analysis was carried out for exogenous variables BF, BV, MTT, bGS, and presurgical biopsy-derived combined Gleason grade and endogenous variables sGS, sGG, MVD, and T stage. A receiver operating characteristics analysis was performed to analyze the discriminating performance of CTP parameters and bGS between intermediate- and high-grade tumors. RESULTS The mean perfusion parameters within the prostate tissue were as follows: BF, $39.1 \pm 13.4 \mathrm{~mL} / 100 \mathrm{~mL} \min ; \mathrm{BV}, 4.9 \pm 2.4 \mathrm{~mL} / 100 \mathrm{~mL}$; and MTT, $8.9 \pm 3.7$ seconds. The mean MVD of the tumor tissue was $144.3 \pm 55.6 / \mathrm{mm}$. Computed tomography perfusion parameters and MVD were significantly higher in patients with high-grade tumors compared with those with intermediate-grade tumors $(\mathrm{P}<0.01$ for $\mathrm{BF}, \mathrm{BV}$, and MVD). Only BV and MVD were significantly different among sGS and sGG groups. Moderate correlations were found between BF and sGS (0.38) and between BV and sGS (0.43). Linear relations of BV to sGS and to sGG were found. Blood volume (area under the curve, 0.86) was superior to bGS (area under the curve, 0.75) in discriminating high-grade from intermediate-grade tumors. CONCLUSION Computed tomography perfusion parameters derived by en bloc perfusion of the prostate are higher in high-grade tumors compared with intermediate-grade tumors. Blood flow and BV correlate with the definitive Gleason score. Blood volume predicts high-grade tumors better than does the Gleason score of biopsy specimens. Further studies are needed to determine a potential role for CTP in prostate cancer patients.
\end{abstract}

DOI: https://doi.org/10.1097/RLI.0000000000000055

Posted at the Zurich Open Repository and Archive, University of Zurich

ZORA URL: https://doi.org/10.5167/uzh-98364

Journal Article

Published Version

Originally published at: 
Huellner, Martin W; Pauli, Chantal; Mattei, Agostino; Ross, Steffen; Diebold, Joachim; Vosbeck, Jürg; Allgayer, Bernhard; Strobel, Klaus; Veit-Haibach, Patrick (2014). Assessment of prostate cancer with dynamic contrast-enhanced computed tomography using an en bloc approach. Investigative Radiology, 49(9):571-578.

DOI: https://doi.org/10.1097/RLI.0000000000000055 


\title{
Assessment of Prostate Cancer With Dynamic Contrast-Enhanced Computed Tomography Using an En Bloc Approach
}

\author{
Martin W. Huellner, MD, * Chantal Pauli, MD, † Agostino Mattei, MD, $\neq$ Steffen Ross, MD, * \\ Joachim Diebold, MD, † Jürg Vosbeck, MD, † Bernhard Allgayer, MD, * Klaus Strobel, MD,* \\ and Patrick Veit-Haibach, MD*
}

\begin{abstract}
Objectives: The aim of this study was to assess the performance of dynamic contrast-enhanced computed tomography of the prostate in patients with biopsy-proven prostate cancer.

Material and Methods: A total of 46 male patients (median age, 65 years; range, 49-73 years) with biopsy-proven prostate cancer underwent an en bloc computed tomography perfusion (CTP) scan of the prostate before surgery. The perfusion parameters mean transit time (MTT), blood flow (BF), and blood volume (BV), as well as the microvessel density (MVD) of surgical specimens were determined. Differences in CTP parameters and MVD among postsurgical Gleason score (sGS) and postsurgical combined Gleason grade (sGG) groups were analyzed. Spearman correlation coefficients were determined between CTP parameters and presurgical biopsy-derived Gleason score (bGS), presurgical biopsy-derived combined Gleason grade (bGG), sGS, sGG, MVD, and pathological tumor stage. A linear regression analysis was carried out for exogenous variables BF, BV, MTT, bGS, and presurgical biopsy-derived combined Gleason grade and endogenous variables sGS, sGG, MVD, and T stage. A receiver operating characteristics analysis was performed to analyze the discriminating performance of CTP parameters and bGS between intermediateand high-grade tumors.
\end{abstract}

Results: The mean perfusion parameters within the prostate tissue were as follows: BF, $39.1 \pm 13.4 \mathrm{~mL} / 100 \mathrm{~mL} \mathrm{~min}^{-1}$; BV, $4.9 \pm 2.4 \mathrm{~mL} / 100 \mathrm{~mL}$; and MTT, $8.9 \pm 3.7$ seconds. The mean MVD of the tumor tissue was $144.3 \pm 55.6 / \mathrm{mm}^{2}$. Computed tomography perfusion parameters and MVD were significantly higher in patients with high-grade tumors compared with those with intermediate-grade tumors ( $P<0.01$ for BF, BV, and MVD). Only BV and MVD were significantly different among sGS and sGG groups. Moderate correlations were found between BF and sGS (0.38) and between BV and sGS (0.43). Linear relations of $\mathrm{BV}$ to sGS and to sGG were found. Blood volume (area under the curve, 0.86 ) was superior to bGS (area under the curve, 0.75) in discriminating high-grade from intermediate-grade tumors.

Conclusion: Computed tomography perfusion parameters derived by en bloc perfusion of the prostate are higher in high-grade tumors compared with intermediate-grade tumors. Blood flow and BV correlate with the definitive Gleason score. Blood volume predicts high-grade tumors better than does the Gleason score of biopsy specimens. Further studies are needed to determine a potential role for CTP in prostate cancer patients.

Received for publication December 24, 2013; and accepted for publication, after revision, February 14, 2014.

From the *Department of Radiology and Nuclear Medicine, $\dagger$ Institute of Pathology, and tDepartment of Urology, Lucerne Cantonal Hospital, Switzerland.

Conflicts of interest and sources of funding: P.V.-H. received IIS grants from Bayer Healthcare and Siemens Healthcare. All other authors declare that they have no conflicts of interests.

Reprints: Martin Huellner, MD, Department of Medical Radiology, Divisions of Nuclear Medicine and Neuroradiology, University Hospital Zurich,

Rämistrasse 100, 8091 Zurich, Switzerland. E-mail: martin.huellner@usz.ch. Copyright (C) 2014 by Lippincott Williams \& Wilkins

ISSN: 0020-9996/14/4909-0571
Key Words: perfusion imaging, computed tomography, prostate cancer, Gleason score, oncology, multimodal imaging

(Invest Radiol 2014;49: 571-578)

$P_{n=1}^{n}$ rostate cancer is the most frequent noncutaneous malignancy in men in developed countries, with an annual incidence of $0.062 \%{ }^{1}$ Its comparably low mortality is attributed to an overall early detection, to varying tumor behaviors from almost benign to rare highly aggressive subtypes, as well as to several effective therapeutic options.

If prostate cancer is suspected in a patient, the work-up usually includes biopsies guided by transrectal ultrasound (TRUS) to confirm the tumor and provide a tentative estimate of its aggressiveness, which will have implications on further therapeutic decisions. It is, however, well known that the presurgical and postsurgical histopathological results, that is, the Gleason grading, may differ considerably. ${ }^{2-4}$

To noninvasively obtain further information about the tumor's aggressiveness before therapy, magnetic resonance imaging (MRI) has gained widespread acceptance during the last decade. Functional methods such as dynamic contrast-enhanced (DCE) MRI, diffusionweighted imaging, and magnetic resonance spectroscopy are used to locate the tumor within the prostate and enable targeted biopsy. ${ }^{5-14}$ It was recently shown that multiparametric MRI is precise in detecting even low-risk prostate cancer, with the accuracy depending on tumor volume and Gleason grade. ${ }^{9}$

Dynamic contrast-enhanced computed tomography (CT) has demonstrated its ability for the noninvasive assessment of angiogenesis by determining functional parameters of tissue microcirculation in different malignant lesions. ${ }^{15-21}$ There are also potential roles for DCE $\mathrm{CT}$ in prostate cancer, such as the initial tumor assessment, follow-up, and monitoring of therapy response in patients with contraindications to MRI, such as pacemakers or ferromagnetic vascular coils. ${ }^{9,22-25}$ The prostate, however, is comparably difficult to assess with CT-perfusion (CTP) because of artifacts from neighboring pelvic bones and the usually small volume of prostate cancer as well as coexisting benign prostatic hyperplasia and chronic prostatitis. On the other hand, several tumor spots of varying grades may coexist or even diffusely infiltrate the prostate parenchyma rather than form circumscribed compounds. The few available studies about CTP in prostate cancer have partly contradictory results concerning both its feasibility and its usefulness. ${ }^{22,23,26-28}$ Korporaal and coworkers ${ }^{28}$ provided evidence that the results of DCE CT are not substantially influenced by the choice of the tracer kinetic model. One recent feasibility study addressed the relationship of image noise and voxel size in DCE CT of the prostate but provided no histopathological information. ${ }^{11}$ Another recent study investigating histopathological correlation found a relationship between blood volume (BV) and microvessel density (MVD). However, there are yet no data available about the association of perfusion parameters derived from DCE CT and clinically relevant histopathological grading such as the Gleason score (GS). 
Thus, the aims of the present study were to assess the whole prostate with DCE CT using an en bloc approach and to analyze the relationship between perfusion parameters and histopathological grading.

\section{MATERIAL AND METHODS}

\section{Patients}

This prospective study was approved by the institutional review board and by the cantonal ethics committee. All patients provided informed signed consent before the examinations.

To be included into the study, patients had to meet the following criteria: biopsy-proven prostate cancer within the last 8 months, referral for initial staging examination by abdominal CT and/or bone scan, willingness to undergo an additional CT scan to acquire DCE image data sets, and a scheduled start of therapy in less than 5 months. The exclusion criteria were renal insufficiency (renal clearance $<30 \mathrm{~mL} / \mathrm{min}$ ) without dialysis, known allergy or hypersensitivity to iodinated contrast medium, and untreated hyperthyroidism. Ninety-eight male patients (median age, 67 years; range, 49-84 years) were finally included and underwent the CTP scan.

Thereof, 18 patients $(18 \%)$ were excluded after the scan because of technical reasons such as power injector malfunction (7 patients), movement artifacts (4 patients), and beam hardening artifacts due to hip prosthesis ( 5 patients) or metal markers within the prostate ( 2 patients). Thirty-two patients $(32 \%)$ were excluded afterward because they did not undergo prostatectomy, making definitive histopathological Gleason grading unavailable. Of these, 2 patients $(3 \%)$ were treated palliatively by transurethral resection of the prostate. Fourteen patients $(14 \%)$ received external beam radiation therapy, 4 patients $(5 \%)$ underwent lowdose brachytherapy with seeds, and 5 patients $(6 \%)$ were treated with palliative antiandrogen drugs. A watchful waiting strategy was chosen in 4 patients $(5 \%)$. Three patients $(4 \%)$ refrained from therapy against medical advice. Two patients (3\%) were excluded because the time interval between $\mathrm{CT}$ scan and prostatectomy was more than 150 days.

The remaining 46 patients were treated by curative surgery (38 by robot-assisted laparoscopic radical prostatectomy and 8 by open radical prostatectomy) and qualified for analysis. The median time interval between biopsy and CT scan was 19 days (range, 0-202 days). The median time interval between the CTP scan and prostatectomy was 63 days (range, 10-147 days). Median time between biopsy and prostatectomy was 83 days (range, 19-244 days).

The presurgical (bGS) and postsurgical (sGS) GS, the presurgical (bGG) and postsurgical (sGG) combined Gleason grade (GG), and pathological tumor stage were available for all 46 patients. Sufficient tissue material for MVD assessment was available for 32 patients. The term combined Gleason grade (GG) was used to characterize the 2 Gleason patterns (eg, bGG $7=4+3$ ), whereas the usual GS refers to the sum of the 2 patterns (eg, bGS 7). It is known that, for example, $7=4+3$ tumors may exhibit more aggressive characteristics than $7=3+$ 4 tumors. $^{29-31}$ Low-grade tumors are defined as a GS of 6 or lower, intermediate tumors correspond to a GS of 7, and high-grade tumors entail a GS of 8 or higher. A detailed overview of patient data is given in Table 1.

\section{DCE-CT Scan}

All CT scans were performed using a Somatom Definition Flash scanner (Siemens Healthcare, Erlangen, Germany). Depending on whether patients were referred for staging CT or bone scan, they underwent a CTP that was integrated into the abdominal staging CT scan using a split-injection protocol or a CTP scan only. The covered scan length of the perfusion scan was $7 \mathrm{~cm}$. The duration of the CTP scan was 60 seconds, with a rotation time of 1 second, corresponding to a sampling interval of 1 second. With such a rather lengthy protocol, high reproducibility of perfusion parameters can be obtained. ${ }^{32}$ The tube current was set to $100 \mathrm{mAs}$, and the tube voltage, to $100 \mathrm{kVp}$ (fixed parameters). Scanning started with a delay of 10 seconds after the injection of $40 \mathrm{~mL}$ of iodinated contrast medium (Ultravist 370; Bayer Healthcare, Leverkusen, Germany), followed by a flush of $50 \mathrm{~mL}$ of $\mathrm{NaCl}$ at $4.5 \mathrm{~mL} / \mathrm{s}$. The contrast agent was injected into an antecubital vein using a dual-head pump injection device (Stellant D; Medrad, Warrendale, PA). The slice collimation was $64 \times 0.6$. The CTP reconstruction increment was $3 \mathrm{~mm}$ at $5-\mathrm{mm}$ slice width. The image reconstruction was performed with a $512 \times 512$-pixel matrix.

\section{Image Evaluation}

All evaluations were performed by 2 experienced radiologists in consensus. The perfusion parameters blood flow (BF), BV, and mean transit time (MTT) were determined by postprocessing on a commercially available computer workstation using a dedicated perfusion assessment software (syngo VPCT Body; Siemens Healthcare). A data set motion correction and a noise reduction algorithm were applied automatically. The processing thresholds were set to 0 and $150 \mathrm{HU}$ to exclude bone and other hyperdense material. The window width

TABLE 1. Patient Data

\begin{tabular}{|c|c|}
\hline Parameter & $\mathbf{n}$ \\
\hline No. patients & 46 \\
\hline Age, median (range), y & $65(49-73)$ \\
\hline PSA, median (range), $\mu \mathrm{g} / \mathrm{L}$ & $8.7(2.6-82.4)$ \\
\hline \multicolumn{2}{|l|}{ bGS } \\
\hline 6 & 9 \\
\hline 7 & 30 \\
\hline 8 & 5 \\
\hline 9 & 2 \\
\hline \multicolumn{2}{|l|}{ bGG } \\
\hline $6(3+3)$ & 9 \\
\hline $7(3+4)$ & 20 \\
\hline $7(4+3)$ & 10 \\
\hline $8(4+4)$ & 4 \\
\hline $8(5+3)$ & 1 \\
\hline $9(4+5)$ & 1 \\
\hline $9(5+4)$ & 1 \\
\hline \multicolumn{2}{|l|}{ sGS } \\
\hline 6 & 1 \\
\hline 7 & 36 \\
\hline 8 & 6 \\
\hline 9 & 3 \\
\hline \multicolumn{2}{|l|}{ sGG } \\
\hline $6(3+3)$ & 1 \\
\hline $7(3+4)$ & 20 \\
\hline $7(4+3)$ & 16 \\
\hline $8(4+4)$ & 5 \\
\hline $8(5+3)$ & 1 \\
\hline $9(4+5)$ & 3 \\
\hline $9(5+4)$ & 0 \\
\hline \multicolumn{2}{|l|}{ Pathological tumor stage } \\
\hline pT2a & 2 \\
\hline pT2b & 0 \\
\hline pT2c & 34 \\
\hline pT3a & 6 \\
\hline pT3b & 4 \\
\hline
\end{tabular}

PSA indicates prostate-specific antigen; bGS, Gleason score of biopsy specimens; bGG, combined Gleason grade of biopsy specimens; sGG, combined Gleason grade of surgical specimens; sGS, Gleason score of surgical specimens. 
and center for reference vessel input were 300 and $150 \mathrm{HU}$, respectively. The relative threshold for inside and outside was $50 \%$. An adaptive smoothing filter was applied. The vendor's default algorithmic parameters were applied.

For every patient, an individual arterial input fraction was determined by placing an analytic region of interest into the external iliac artery to obtain a time attenuation curve. This indirect method was chosen because the internal iliac artery, which supplies the prostate, was not depicted adequately within the scan range in all patients. It was presumed that the time attenuation curves derived from both arteries were similar.

Three-dimensional color-coded maps of BF, BV, and MTT were generated with a sequential 2-compartment model (modified Patlak approach). Blood flow is defined as the amount of blood flowing through $100 \mathrm{~mL}$ of prostate tissue within 1 minute. Mean transit time is defined as the average time of contrast agent residence within the prostate tissue. Blood volume is the product of BF and MTT and is defined as the amount of blood within $100 \mathrm{~mL}$ of prostate tissue. Blood volume can be expressed as proportion of the total volume of a dedicated voxel.

The perfusion parameter values of the prostate were determined using an en bloc approach. For this purpose, an analytic volume of interest was placed on the prostate on the time-maximum intensity projection images, which was simultaneously transferred to the spatially coregistered color-coded data sets by software automatism. The contour of the volume of interest was adapted to the contour of the prostate on all time-maximum intensity projection images, in all 3 planes.

\section{Histopathological Analysis}

The GS of biopsy specimens and prostatectomy specimens as well as tumor stage of prostatectomy specimens were determined according to internationally standardized methods in pathology. ${ }^{33,34}$

For the characterization of the tumor vasculature, the mean intratumoral MVD was quantitatively assessed according to immunohistochemical cluster of differentiation molecule 34 (CD34) staining. Formalin-fixed prostatectomy specimens embedded in paraffin could be obtained from 32 patients. In the remaining 14 patients, the amount of material that was left after standard histopathological examination was too small to allow for MVD analysis. Specimens were cut into 4- $\mu \mathrm{m}$ slices and fixed to histology slides (x-tra; Leica Biosystems, Nussloch, Germany). After hematoxylin/eosin staining, slides were stained with CD34 antibody (1:30, NCL-L END, Novocastra; Leica Biosystems) using an automated staining system (BenchMark XT; Ventana Medical Systems, Oro Valley, AZ). The slides were then scanned with a slide scanner (iScan Coreo; Ventana Medical Systems). One pathologist blinded to clinical and imaging data performed the histopathological analysis by visually counting the positive microvessels on the scanned images using public domain software (imageJ, http://rsbweb.nih.gov/ijIm Cache).

\section{Statistical Analysis}

Differences in CTP parameters of the prostate and tumoral MVD among groups of tumors with different grading were evaluated using Mann-Whitney $U$ test and/or Kruskal-Wallis test. Correlations between pairs of variables (CTP parameters, MVD, bGS, bGG, sGS, sGG, T stage) were assessed using Spearman rank correlation coefficient. Results were interpreted as strong correlation if between \pm 0.5 and \pm 1.0 , moderate correlation between \pm 0.3 and \pm 0.49 , and weak correlation between \pm 0.1 and \pm 0.29 , thereunder no correlation. ${ }^{35} \mathrm{~A}$ linear regression analysis was performed to assess the relationship of exogenous variables BF, BV, MTT, bGS, and bGG and endogenous variables sGS, sGG, MVD, and T stage. A receiver operating characteristics (ROC) analysis was carried out to analyze the discriminating performance of CTP parameters and bGS between intermediate- and high-grade tumors. A $P$ value of $<0.05$ was considered statistically significant. All analyses were performed using the software IBM SPSS Statistics 19.0.1 (SPSS Inc, Chicago, IL).

\section{RESULTS}

\section{Histopathology}

In both biopsy and prostatectomy specimens, the most common GS was 7 (range, 6-9) and the most common GG was $7(3+4)$. Histopathological upgrading from biopsy to prostatectomy specimens occurred in 12 patients, and downgrading, in 4 patients. A change in the GG occurred in 26 patients ( $56 \%$; range, -1 to +4 grades). Whereas there were initially 9 patients diagnosed with bGS 6 , there was only 1 patient left with sGS 6 . The most common first Gleason pattern in biopsy samples was 3 , whereas the most common first pattern was 4 in surgical samples. According to the final histopathological results, there was 1 low-grade tumor ( $2 \%$, sGS 6), 36 intermediate-grade tumors (78\%, sGS 7), and 9 high-grade tumors (20\%, sGS 8 and 9).

The most common tumor stage was pT2c (34 patients, 74\%), denoting invasion of both lobes of the prostate. Extracapsular spread (T3) occurred in 10 patients $(22 \%)$, thereof invasion of the seminal vesicles (T3b) in 4 patients $(9 \%)$. The mean MVD was $144.3 \pm 55.6$ microvessels $/ \mathrm{mm}^{2}$.

An overview of relevant data is given in Table 1.

\section{DCE-CT and Statistical Analyses}

The calculated mean effective individual radiation dose was $25.6 \mathrm{mSv}$. The BF in prostate tissue was $39.1 \pm 13.4 \mathrm{~mL} / 100 \mathrm{~mL} \mathrm{~min}^{-1}$, the mean BV was $4.9 \pm 2.4 \mathrm{~mL} / 100 \mathrm{~mL}$, and the mean MTT was $8.9 \pm$ 3.7 seconds. A pictorial example is given in Figure 1. Both CTP parameters and MVD were significantly higher in patients with highgrade tumors compared with those with intermediate-grade tumors $(P<0.01$ for BF, BV, and MVD). When broken down further into sGS and $\mathrm{sGG}$ groups, only the differences in BV and MVD remained at
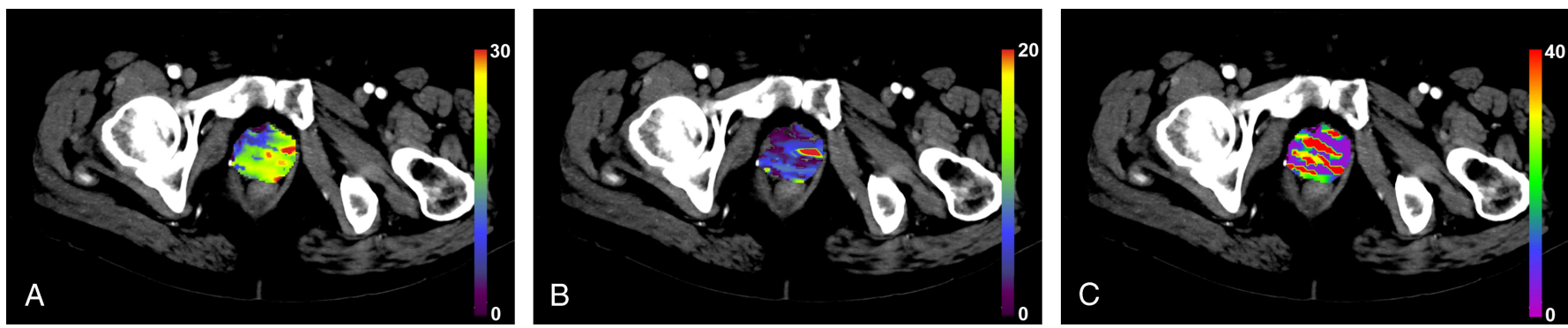

FIGURE 1. Patient with prostate cancer and GS $7(3+4)$. Color-coded maps of perfusion parameters $\mathrm{BF}\left(28.5 \mathrm{~mL} / 100 \mathrm{~mL} \mathrm{~min}^{-1}\right)$ (A), BV (4.1 mL/100 mL) (B), and MTT (8.1 seconds) (C) are shown. 
TABLE 2. CT-Perfusion Parameters and Microvessel Density Stratified by Tumor Aggressiveness

\begin{tabular}{|c|c|c|c|c|c|c|c|c|c|c|c|c|c|c|c|}
\hline & \multicolumn{15}{|c|}{ Perfusion Parameters } \\
\hline & $\mathbf{n}$ & $\begin{array}{c}\text { BF } \\
\text { Mean } \pm \text { SD, } \\
\text { mL/100 } \mathrm{mL} \mathrm{min}^{-1}\end{array}$ & & & $P$ & & & & $\begin{array}{c}\text { BV } \\
\text { Mean } \pm \text { SD, } \\
\mathrm{mL} / 100 \mathrm{~mL}\end{array}$ & & & $P$ & & & \\
\hline All patients & 46 & $39.1 \pm 13.4$ & & & & & & & $4.9 \pm 2.4$ & & & & & & \\
\hline $\begin{array}{l}\text { Intermediate-grade } \\
\text { tumor patients } \\
\text { (sGS 7) }\end{array}$ & 36 & $36.4 \pm 12.3$ & & & $<0.01$ & & & & $4.2 \pm 1.6$ & & & $<0.001$ & & & \\
\hline $\begin{array}{l}\text { High-grade } \\
\text { tumor patients } \\
(\mathrm{sGS}>7)\end{array}$ & 9 & $50.5 \pm 12.8$ & & & & & & & $7.6 \pm 3.1$ & & & & & & \\
\hline \multicolumn{16}{|l|}{ sGS } \\
\hline & & & 6 & 7 & 8 & & & 0.06 & & 6 & 7 & 8 & & & $<0.01$ \\
\hline 6 & 1 & 35.4 & N/A & 0.93 & 0.13 & & & & 5.2 & N/A & 0.33 & 0.32 & & & \\
\hline 7 & 36 & $36.4 \pm 12.3$ & 0.93 & N/A & 0.01 & & & & $4.2 \pm 1.6$ & 0.33 & N/A & $<0.01$ & & & \\
\hline 8 & 6 & $50.3 \pm 8.7$ & 0.13 & 0.01 & N/A & & & & $6.5 \pm 1.3$ & 0.32 & $<0.01$ & N/A & & & \\
\hline 9 & 3 & $51.0 \pm 21.5$ & 0.66 & 0.21 & 0.61 & & & & $9.9 \pm 4.9$ & 0.66 & 0.03 & 0.30 & & & \\
\hline \multicolumn{16}{|l|}{ sGG } \\
\hline & & & $6(3+3)$ & $7(3+4)$ & $7(4+3)$ & $8(4+4)$ & $8(5+3)$ & 0.19 & & $6(3+3)$ & $7(3+4)$ & $7(4+3)$ & $8(4+4)$ & $8(5+3)$ & 0.01 \\
\hline $6(3+3)$ & 1 & 35.4 & N/A & 0.87 & 1.00 & 0.14 & 0.32 & & 5.2 & N/A & 0.41 & 0.26 & 0.14 & 0.32 & \\
\hline $7(3+4)$ & 20 & $37.1 \pm 10.8$ & 0.87 & $\mathrm{~N} / \mathrm{A}$ & 0.70 & 0.04 & 0.19 & & $4.5 \pm 1.4$ & 0.41 & N/A & 0.18 & $<0.01$ & 0.87 & \\
\hline $7(4+3)$ & 16 & $35.4 \pm 14.3$ & 1.00 & 0.70 & N/A & 0.06 & 0.15 & & $3.8 \pm 1.7$ & 0.26 & 0.18 & N/A & $<0.01$ & 0.84 & \\
\hline $8(4+4)$ & 5 & $49.2 \pm 9.2$ & 0.14 & 0.04 & 0.06 & N/A & 0.38 & & $6.9 \pm 0.9$ & 0.14 & $<0.01$ & $<0.01$ & N/A & 0.14 & \\
\hline $8(5+3)$ & 1 & 55.9 & 0.32 & 0.19 & 0.15 & 0.38 & N/A & & 4.3 & 0.32 & 0.87 & 0.84 & 0.14 & N/A & \\
\hline $9(4+5)$ & 3 & $51.0 \pm 21.5$ & 0.66 & 0.24 & 0.22 & 0.66 & 0.66 & & $9.9 \pm 4.9$ & 0.66 & 0.05 & 0.03 & 0.46 & 0.18 & \\
\hline
\end{tabular}

BF indicates blood flow; BV, blood volume; MTT, mean transit time; N/A, not applicable; sGS, Gleason score of surgical specimens; sGG, combined Gleason grade of surgical specimens.

a statistically significant level among these groups, with overall higher values in higher sGS and sGG tumors. Detailed results are provided in Table 2 .

Moderate correlations were found between BF and sGS $(0.38$; $P<0.01)$ and between BV and sGS $(0.43 ; P<0.01)$. Only weak to moderate correlations of borderline significance were found between $\mathrm{BV}$ and bGS as well as bGG. No correlation was observed between perfusion parameters and MVD as well as T stage (Table 3).

The regression analysis showed linear relationships of BV to sGS (correlation coefficient of determination $\left[r^{2}\right]=0.37$; Fig. $2 \mathrm{~A}$ ) as well as to $\mathrm{sGG}\left(r^{2}=0.25\right.$; Fig. $\left.2 \mathrm{~A}\right)$. No valuable linear relations were found between perfusion parameters and $\mathrm{T}$ stage as well as MVD (Table 4).

The ROC analysis revealed that BV (area under the curve [AUC], 0.86; $P=0.001$ ) was superior to BF (AUC, $0.79 ; P=0.003$ ) as well as to bGS (AUC, $0.75 ; P=0.02$ ) in discriminating high-grade tumors from intermediate-grade tumors (Fig. 3).

\section{DISCUSSION}

Oncologic perfusion imaging relies on functional and structural interference in tissue induced by a tumor. ${ }^{36}$ So far, CTP has been used in a variety of neoplasms, including head and neck cancer, lung cancer, colorectal cancer, and hepatocellular carcinoma. ${ }^{17-19,37-39}$ However, there are only a few studies available investigating CTP in prostate cancer, and in part, they show contradictory results concerning its feasibility and usefulness. ${ }^{22,23,26-28}$

\section{En Bloc Approach}

There are several factors that challenge CTP in this glandular organ. The lack of contrast resolution makes it impossible to visually localize cancer on nonenhanced $\mathrm{CT} .^{13,23}$ This challenged the centering of the perfusion volume in older studies at times when only z-axis coverage of less than $4 \mathrm{~cm}$ was available. ${ }^{22,23,26,27}$ But even with today's 256-slice scanners that allow for a much larger z-axis (up to $28 \mathrm{~cm}$ ), it is still almost impossible to localize prostate cancer on contrast-enhanced CT images in the absence of extracapsular spread. This limits a retrospective approach to CTP, although the whole prostate can now be covered easily. Furthermore, neighboring pelvic bones cause streak artifacts and beam-hardening artifacts on CT images,

TABLE 3. Spearman Correlation Coefficients $(r)$ of CT-Perfusion Parameters and Gleason Parameters, T Stage, and MVD

\begin{tabular}{|c|c|c|c|c|c|c|}
\hline & \multicolumn{2}{|c|}{ BF } & \multicolumn{2}{|c|}{ BV } & \multicolumn{2}{|c|}{ MTT } \\
\hline & $r$ & $P$ & $r$ & $P$ & $r$ & $P$ \\
\hline bGS & 0.27 & 0.07 & 0.30 & 0.04 & 0.13 & 0.39 \\
\hline bGG & 0.26 & 0.08 & 0.29 & 0.05 & 0.15 & 0.32 \\
\hline sGS & 0.38 & $<0.01$ & 0.43 & $<0.01$ & 0.30 & 0.04 \\
\hline sGG & 0.27 & 0.07 & 0.22 & 0.14 & 0.22 & 0.15 \\
\hline $\mathrm{T}$ stage & -0.01 & 0.96 & 0.13 & 0.40 & 0.18 & 0.22 \\
\hline MVD* & 0.26 & 0.16 & 0.18 & 0.33 & 0.16 & 0.38 \\
\hline
\end{tabular}

$\mathrm{BF}$ indicates blood flow; BV, blood volume; MTT, mean transit time; bGS, Gleason score of biopsy specimens; bGG, combined Gleason grade of biopsy specimens; sGS, Gleason score of surgical specimens; sGG, combined Gleason grade of surgical specimens; MVD, microvessel density.

*Data available from 32 subjects only ( 25 intermediate-grade tumor patients, 7 high-grade tumor patients). 
Microvessel Density, $1 / \mathrm{mm}^{2}$

MTT

\begin{tabular}{|c|c|c|c|c|c|c|c|c|c|c|}
\hline Mean \pm SD, & & & $P$ & & $n$ & & & & $P$ & \\
\hline $8.9 \pm 3.7$ & & & & & 32 & $144.3 \pm 55.6$ & & & & \\
\hline $10.7 \pm 3.2$ & & & & & 7 & $204.1 \pm 58.5$ & & & & \\
\hline & 6 & 7 & 8 & 0.06 & & & 6 & 7 & 8 & 0.02 \\
\hline 9.5 & N/A & 0.35 & 0.62 & & 0 & N/A & N/A & N/A & N/A & \\
\hline $8.5 \pm 3.8$ & 0.35 & N/A & 0.25 & & 25 & $127.6 \pm 42.4$ & N/A & N/A & 0.03 & \\
\hline $9.3 \pm 2.2$ & 0.62 & 0.25 & N/A & & 4 & $213.5 \pm 70.1$ & N/A & 0.03 & N/A & \\
\hline $13.6 \pm 3.1$ & 0.18 & 0.02 & 0.20 & & 3 & $191.6 \pm 49.8$ & N/A & 0.05 & 0.72 & \\
\hline
\end{tabular}

\begin{tabular}{|c|c|c|c|c|c|c|c|c|c|c|c|c|}
\hline & $6(3+3)$ & $7(3+4)$ & $7(4+3)$ & $8(4+4)$ & $8(5+3)$ & 0.08 & & $6(3+3)$ & $7(3+4)$ & $7(4+3)$ & $8(4+4)$ & $\mathbf{8}(\mathbf{5}+\mathbf{3})<0.01$ \\
\hline 9.5 & N/A & 0.51 & 0.22 & 0.38 & 0.32 & 0 & N/A & N/A & N/A & N/A & N/A & N/A \\
\hline $9.0 \pm 4.7$ & 0.51 & N/A & 0.89 & 0.15 & 0.32 & 13 & $107.9 \pm 45.7$ & N/A & N/A & 0.02 & 0.01 & N/A \\
\hline $7.8 \pm 2.0$ & 0.22 & 0.89 & N/A & 0.07 & 0.31 & 12 & $148.9 \pm 26.5$ & N/A & 0.02 & $\mathrm{~N} / \mathrm{A}$ & 0.12 & N/A \\
\hline $9.9 \pm 1.8$ & 0.38 & 0.15 & 0.07 & N/A & 0.14 & 4 & $213.5 \pm 70.1$ & N/A & 0.01 & 0.12 & N/A & N/A \\
\hline 6.2 & 0.32 & 0.32 & 0.31 & 0.14 & N/A & 0 & N/A & N/A & N/A & N/A & N/A & N/A \\
\hline $13.6 \pm 3.1$ & 0.18 & 0.05 & 0.01 & 0.03 & 0.18 & 3 & $191.6 \pm 49.8$ & N/A & 0.04 & 0.11 & 0.72 & N/A \\
\hline
\end{tabular}

which might degrade perfusion data. Thus, the standard approach in CTP imaging, selectively analyzing areas within the organ that correspond to tumor, is compromised. Our rationale in the present study was therefore to use an en bloc approach.

\section{Comparison With Histopathology}

Our study represents the first systematic evaluation of en bloc DCE CT in patients with prostate cancer and 1 of the first studies to address the relationship between CTP parameters and histopathological grading. A direct comparison of perfusion data with whole-mount histopathological specimens would be a desirable way to clarify if CTP imaging is valuable in prostate cancer. However, because of the small size of the prostate and of the limited spatial resolution of parametric maps derived from CTP, this comparison is challenging. ${ }^{23}$ Furthermore, prostate cancer is multifocal in most patients, with different sites often exhibiting different histological grades. Osimani and coworkers $^{23}$ found a correlation between BV and MVD. Their BV in fields with prostate cancer was $8.45 \pm 2.75 \mathrm{~mL} / 100 \mathrm{~mL}$; however, there was considerable overlap in $\mathrm{BV}$ values of fields with prostate cancer, benign hyperplasia, and healthy tissue. ${ }^{23}$ In our study, we found no significant correlation of perfusion parameters with MVD. This might be due to our en bloc approach, which might blur such a relationship between tumor-derived MVD and whole-prostate-derived perfusion parameters. Other explanations might be the rather small
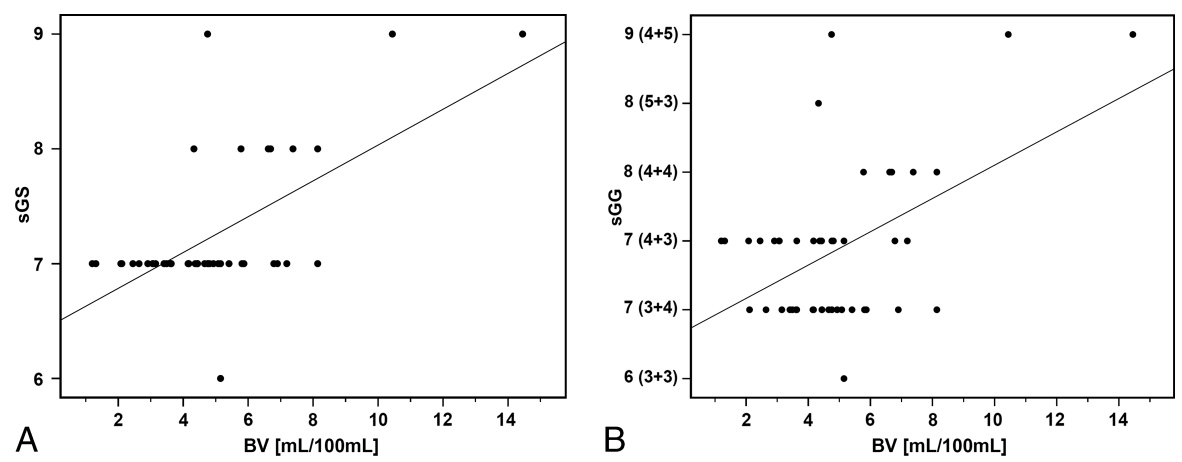

FIGURE 2. Linear regression analysis of BV and $\operatorname{sGS}\left(r^{2}=0.37\right)(\mathrm{A})$ and $\mathrm{BV}$ and $\mathrm{sGG}\left(r^{2}=0.25\right)(\mathrm{B}) . r^{2}$ indicates regression coefficient of determination. 
TABLE 4. Linear Regression Coefficients of Determination $\left(r^{2}\right)$ of Postsurgical Histopathological Results

\begin{tabular}{lccccc}
\hline \multirow{2}{*}{$\begin{array}{l}\text { Endogenous } \\
\text { Variables }\end{array}$} & \multicolumn{5}{c}{ Exogenous Variables } \\
\cline { 2 - 6 } & BF & BV & MTT & bGS & bGG \\
\hline sGS & 0.15 & 0.37 & 0.09 & 0.23 & 0.29 \\
SGG & 0.12 & 0.25 & 0.04 & 0.13 & 0.14 \\
T stage & 0.01 & 0.01 & 0.01 & 0.13 & 0.14 \\
MVD* & 0.07 & 0.10 & 0.01 & 0.05 & 0.06 \\
\hline
\end{tabular}

$\mathrm{BF}$ indicates blood flow; BV, blood volume; MTT, mean transit time; bGS, Gleason score of biopsy specimens; bGG, combined Gleason grade of biopsy specimens; sGS, Gleason score of surgical specimens; sGG, combined Gleason grade of surgical specimens; MVD, microvessel density.

*Data available from 32 subjects only.

number of subjects with MVD data available or the use of CD34 staining for MVD analysis, which might be inferior to CD31 staining. ${ }^{40}$ Besides, it was also shown in recent magnetic resonance (MR) studies that BV does not consistently correlate with histological parameters. ${ }^{40,41}$ However, we found a moderate correlation of the perfusion parameters $\mathrm{BF}$ and $\mathrm{BV}$ with the GS, notably a clinically more relevant parameter than MVD.

We could also demonstrate a linearly directed relationship between the GS and BF as well as BV. Osimani and coworkers did not provide data on the association of DCE CT parameters and histological tumor grade because they included only patients with a GS of 7. In the present study, we found higher $\mathrm{BF}$ and $\mathrm{BV}$ in patients with high-grade tumors $(\mathrm{GS}>7)$ compared with patients with intermediate-grade tumors (GS 7). The higher the GS (sGS) and the GG (sGG), the higher $\mathrm{BV}$ was by trend. This suggests that the correlation of BV and MVD that was proposed by Osimani et al might be reflected by a higher BV in high-grade tumors, which survives through a rather coarse approach such as en bloc DCE analysis. Thus, we suppose that the decreasing tumor differentiation from low to high GS might be reflected by a reciprocal increase in perfusion parameters $\mathrm{BV}$ and $\mathrm{BF}$, possibly because of increased tumor vascularity - at least concerning intermediate- and high-grade tumors - whereas our data do not allow assumption of such a relationship for low-grade tumors. With DCE MR studies, it was shown that quantitative (transfer constant) and semiquantitative (wash-in gradient, wash-out gradient) parameters of prostate cancer tissue are correlated with the GS. ${ }^{25,42}$

\section{Discrimination of Tumor Grades}

Using an ROC analysis, we were also able to show that BV derived from the en bloc perfusion method was a better diagnostic test than was the biopsy GS for the differentiation of intermediate-grade from high-grade tumors. Thus, the tumor aggressiveness might be predicted more accurately by BV than by the GS of biopsy specimens. With TRUS-guided biopsy serving as standard of reference, Chen et $\mathrm{al}^{25}$ showed that the wash-out gradient derived by DCE MR is also able to differentiate between aggressive and less aggressive histological subtypes of prostate cancer. Vos and coworkers ${ }^{42}$ demonstrated that lowgrade peripheral zone tumors can be discriminated from intermediateand high-grade peripheral zone tumors by transfer constant, rate constant (AUC, 0.72), wash-in gradient, and wash-out gradient from DCE MR, with prostatectomy serving as standard of reference. It is known that the GSs of biopsy and prostatectomy specimens might differ considerably, which is caused by unrepresentative sampling, and not to a real change. Upgrading of the GS, as in the present study, has been observed in several previous studies. ${ }^{2-4}$ However, we believe that the performance of TRUS-guided biopsy in our study cohort was still comparably good, given the linear relationship of sGS and bGG $\left(r^{2}=0.29\right)$.

\section{Tumor Stage}

Being reflected by a higher GS, aggressive tumors are associated with a more advanced tumor stage than less aggressive tumors are. ${ }^{31,43}$ In the present study, we found no significant correlation between perfusion parameters and tumor stage. This is probably related to the large portion of subjects $(74 \%)$ in the same tumor stage (pT2c) and is supported by the fact that we found no significant correlation between T stage and sGS $(r=0.22, P=0.14)$. Analogously, Ives and coworkers ${ }^{26}$ found a significant correlation of DCE CT parameters and tumor size only in 2 of 10 subjects $(20 \%)$ with high-grade tumors.

\section{Clinical Implementation and Radiation Burden}

Because a perfusion procedure is readily implemented into a contrast-enhanced CT scan, CTP could potentially serve as an add-on to a pelvic CT scan for prostate cancer staging, besides being less time-consuming than functional MRI. We however acknowledge that CTP suffers from several drawbacks, such as lack of simultaneous high-quality anatomical information within prostate tissue and comparably high radiation burden, and hence will possibly be relevant only for a small population, such as patients with contraindications to MRI or institutions without an MR scanner. However, for these restricted populations, it might be of value to know whether CTP performs better than TRUS-guided biopsy.

Radiation exposure to patients is of growing importance, and MR perfusion, being the competing standard, has a distinct advantage over CTP here. The radiation dose of our protocol is within the range of usual body CTP protocols in oncology. ${ }^{44,45}$ Within the direct $\mathrm{x}$-ray beam in prostatic CTP, the organ that is most vulnerable to radiation is the prostate itself, and no other sensitive organs are contained within the x-ray volume besides the bone marrow. The prostate is usually sought to undergo definitive therapy, either by surgery or by radiation therapy, and unlike in other oncological CTP applications, such as colorectal cancer or lung cancer, no functioning prostate parenchyma is left after therapy. Considering the proximity of the x-ray volume to the gonads, there is, however, the issue of scatter radiation, which might not play a major role in prostate cancer patients, who are usually of an advanced age at diagnosis (median of 67 years in our cohort).

\section{Limitations}

Several limitations have to be accounted for in our study. One major limitation is of course the radiation dose delivered to the

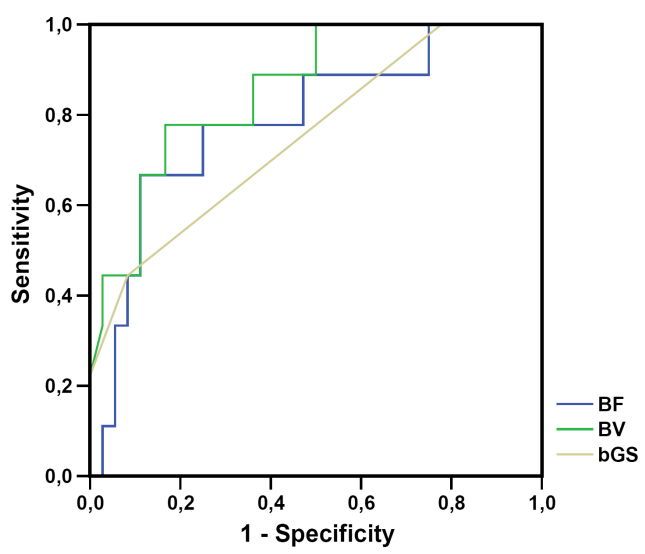

FIGURE 3. ROC curves of the discriminating performance between intermediate- and high-grade cancers. BV (green line; AUC, $0.86 ; \mathrm{Cl}, 0.73-0.99 ; P=0.001$ ) was superior to $\mathrm{BF}$ (blue line) (AUC, 0.79; $\mathrm{Cl}, 0.62-0.96 ; P=0.003$ ) and bGS (beige line) (AUC, $0.75 ; \mathrm{Cl}, 0.57-0.93 ; P=0.02) . \mathrm{Cl}$ indicates confidence interval. 
patient, with the effective dose being comparatively higher than that of a standard pelvic CT. Another limitation is the time interval between CTP examination and prostatectomy (median of 63 days). Tumors might change during that period. However, prostate cancer usually is of a slow-growing nature, and thereby, a degradation of results is unlikely. Despite the initial inclusion of 98 patients, there were MVD data available in only 32 subjects, which might have contributed to the lack of correlation with CTP parameters. Another major limitation is that there was only a single low-grade tumor in our cohort, which limits our results to intermediate- and high-grade tumors. This is certainly a selection bias because low-grade tumors are usually not treated by prostatectomy. This limitation is particularly important because low-grade tumors might undergo active surveillance, and all other tumors are treated in some way in most patients. Blood flow, BV, and MTT provide information only on perfusion and not on permeability of vessels and presumed contrast leakage in tumoral tissue. We also did not account for benign prostatic hyperplasia, necrosis, fibrosis, chronic prostatitis, and other factors that might have an influence on the perfusion parameters.

\section{CONCLUSION}

This is the first study to demonstrate the relationship between DCE CT parameters derived from an en bloc approach and histopathological grading in prostate cancer. It may be considered a first step to clarify if DCE CT parameters can give pertinent information related to histological benchmarks such as the GS. We found that BV is different among different tumor grades and predicts high-grade tumors better than the GS of biopsy specimens does. Further studies with a more specific approach to perfusion imaging and histopathology are needed to define a possible value of DCE CT in prostate cancer patients.

\section{ACKNOWLEDGMENTS}

This work would not have been possible without the invaluable help of our CT and nuclear medicine technicians: Arion Bucher, Mirjam Debon, Judith Ettlin, Mathias Hertl, Jürgen Krüsel, Savo Matijasevic, Gabriella Pasquini, Christiane Wittwer, and Nicole Zülch.

\section{REFERENCES}

1. Jemal A, Bray F, Center MM, et al. Global cancer statistics. CA Cancer J Clin. 2011;61:69-90.

2. Djavan B, Kadesky K, Klopukh B, et al. Gleason scores from prostate biopsies obtained with 18-gauge biopsy needles poorly predict Gleason scores of radical prostatectomy specimens. Eur Urol. 1998;33:261-270.

3. Nayyar R, Singh P, Gupta NP, et al. Upgrading of Gleason score on radical prostatectomy specimen compared to the pre-operative needle core biopsy: an Indian experience. Indian J Urol. 2010;26:56-59.

4. Zam NA, Tan PH, Sim HG, et al. Correlation between prostate needle biopsies and radical prostatectomy specimens: can we predict pathological outcome? Pathology. 2008;40:586-591.

5. Bonekamp D, Jacobs MA, El-Khouli R, et al. Advancements in MR imaging of the prostate: from diagnosis to interventions. Radiographics. 2011;31:677-703.

6. Hoeks CM, Barentsz JO, Hambrock T, et al. Prostate cancer: multiparametric MR imaging for detection, localization, and staging. Radiology. 2011;261:46-66.

7. Mueller-Lisse UG, Miller K. Imaging modalities for primary diagnosis and staging of prostate cancer. Urologe A. 2010;49:190-198.

8. Bittencourt LK, Barentsz JO, de Miranda LC, et al. Prostate MRI: diffusionweighted imaging at $1.5 \mathrm{~T}$ correlates better with prostatectomy Gleason grades than TRUS-guided biopsies in peripheral zone tumours. Eur Radiol. 2012; 22:468-475.

9. Kim JY, Kim SH, Kim YH, et al. Low-risk prostate cancer: the accuracy of multiparametric MR imaging for detection. Radiology. 2014;130801. [Epub ahead of print].

10. Somford DM, Hamoen EH, Futterer JJ, et al. The predictive value of endorectal 3 tesla multiparametric magnetic resonance imaging for extraprostatic extension in patients with low, intermediate and high risk prostate cancer. $J$ Urol. 2013;190:1728-1734.

11. Selnaes KM, Heerschap A, Jensen LR, et al. Peripheral zone prostate cancer localization by multiparametric magnetic resonance at $3 \mathrm{~T}$ : unbiased cancer identification by matching to histopathology. Invest Radiol. 2012;47:624-633.
12. Sertdemir M, Schoenberg SO, Sourbron S, et al. Interscanner comparison of dynamic contrast-enhanced MRI in prostate cancer: 1.5 versus 3 T MRI. Invest Radiol. 2013;48:92-97.

13. Somford DM, Hoeks CM, Hulsbergen-van de Kaa CA, et al. Evaluation of diffusion-weighted MR imaging at inclusion in an active surveillance protocol for low-risk prostate cancer. Invest Radiol. 2013;48:152-157.

14. Somford DM, Hambrock T, Hulsbergen-van de Kaa CA, et al. Initial experience with identifying high-grade prostate cancer using diffusion-weighted MR imaging (DWI) in patients with a Gleason score $</=3+3=6$ upon schematic TRUS-guided biopsy: a radical prostatectomy correlated series. Invest Radiol. 2012;47:153-158.

15. Miles KA. Perfusion CT for the assessment of tumour vascularity: which protocol? Br J Radiol. 2003;76:S36-S42.

16. Miles KA. Functional CT imaging in oncology. Eur Radiol. 2003;13(suppl 5): M134-M138.

17. Goh V, Halligan S, Taylor SA, et al. Differentiation between diverticulitis and colorectal cancer: quantitative CT perfusion measurements versus morphologic criteria - initial experience. Radiology. 2007;242:456-462.

18. Bisdas S, Baghi M, Smolarz A, et al. Quantitative measurements of perfusion and permeability of oropharyngeal and oral cavity cancer, recurrent disease, and associated lymph nodes using first-pass contrast-enhanced computed tomography studies. Invest Radiol. 2007;42:172-179.

19. Veit-Haibach P, Schmid D, Strobel K, et al. Combined PET/CT-perfusion in patients with head and neck cancers. Eur Radiol. 2013;23:163-173.

20. Goh V, Ng QS, Miles K. Computed tomography perfusion imaging for therapeutic assessment: has it come of age as a biomarker in oncology? Invest Radiol. 2012;47:2-4.

21. Reiner CS, Roessle M, Thiesler T, et al. Computed tomography perfusion imaging of renal cell carcinoma: systematic comparison with histopathological angiogenic and prognostic markers. Invest Radiol. 2013;48:183-191.

22. Harvey CJ, Blomley MJ, Dawson P, et al. Functional CT imaging of the acute hyperemic response to radiation therapy of the prostate gland: early experience. J Comput Assist Tomogr. 2001;25:43-49.

23. Osimani M, Bellini D, Di Cristofano C, et al. Perfusion MDCT of prostate cancer: correlation of perfusion $\mathrm{CT}$ parameters and immunohistochemical markers of angiogenesis. AJR Am J Roentgenol. 2012;199:1042-1048.

24. Cyran CC, von Einem JC, Paprottka PM, et al. Dynamic contrast-enhanced computed tomography imaging biomarkers correlated with immunohistochemistry for monitoring the effects of sorafenib on experimental prostate carcinomas. Invest Radiol. 2012;47:49-57.

25. Chen YJ, Chu WC, Pu YS, et al. Washout gradient in dynamic contrastenhanced MRI is associated with tumor aggressiveness of prostate cancer. $J$ Magn Reson Imaging. 2012;36:912-919.

26. Ives EP, Burke MA, Edmonds PR, et al. Quantitative computed tomography perfusion of prostate cancer: correlation with whole-mount pathology. Clin Prostate Cancer. 2005;4:109-112.

27. Win Z, Ariff B, Harvey CJ, et al. Comparative study of experienced vs nonexperienced radiologists in assessing parametric $\mathrm{CT}$ images of the response of the prostate gland to radiotherapy. Br J Radiol. 2008;81:572-576.

28. Korporaal JG, van Vulpen $\mathrm{M}$, van den Berg CA, et al. Tracer kinetic model selection for dynamic contrast-enhanced computed tomography imaging of prostate cancer. Invest Radiol. 2012;47:41-48.

29. Corcoran NM, Hong MK, Casey RG, et al. Upgrade in Gleason score between prostate biopsies and pathology following radical prostatectomy significantly impacts upon the risk of biochemical recurrence. BJU Int. 2011;108:E202-E210.

30. Chan TY, Partin AW, Walsh PC, et al. Prognostic significance of Gleason score $3+4$ versus Gleason score $4+3$ tumor at radical prostatectomy. Urology. 2000;56:823-827.

31. Orozco R, O'Dowd G, Kunnel B, et al. Observations on pathology trends in 62,537 prostate biopsies obtained from urology private practices in the United States. Urology. 1998;51:186-195.

32. Ng CS, Chandler AG, Wei W, et al. Reproducibility of perfusion parameters obtained from perfusion CT in lung tumors. AJR Am J Roentgenol. 2011; 197:113-121

33. Epstein JI, Allsbrook WC Jr, Amin MB, et al. The 2005 international society of urological pathology (ISUP) consensus conference on Gleason grading of prostatic carcinoma. Am J Surg Pathol. 2005;29:1228-1242.

34. Magi-Galluzzi C, Evans AJ, Delahunt B, et al. International society of urological pathology (ISUP) consensus conference on handling and staging of radical prostatectomy specimens. Working group 3: extraprostatic extension, lymphovascular invasion and locally advanced disease. Mod Pathol. 2011;24:26-38.

35. Rodgers JL, Nicewander WA. Thirteen ways to look at the correlation coefficient. Am Stat. 1988;42:59-66.

36. Somford DM, Futterer JJ, Hambrock T, et al. Diffusion and perfusion MR imaging of the prostate. Magn Reson Imaging Clin North Am. 2008; 16:685-695, ix.

37. Miles KA, Griffiths MR, Keith CJ. Blood flow-metabolic relationships are dependent on tumour size in non-small cell lung cancer: a study using quantitative contrast-enhanced computer tomography and positron emission tomography. Eur J Nucl Med Mol Imaging. 2006;33:22-28. 
38. Meijerink MR, van Waesberghe JH, van der Weide L, et al. Early detection of local RFA site recurrence using total liver volume perfusion CT initial experience. Acad Radiol. 2009;16:1215-1222.

39. Veit-Haibach P, Treyer V, Strobel K, et al. Feasibility of integrated CT-liver perfusion in routine FDG-PET/CT. Abdom Imaging. 2010;35:528-536.

40. Oto A, Yang C, Kayhan A, et al. Diffusion-weighted and dynamic contrastenhanced MRI of prostate cancer: correlation of quantitative MR parameters with Gleason score and tumor angiogenesis. AJR Am J Roentgenol. 2011; 197:1382-1390.

41. Franiel T, Ludemann L, Rudolph B, et al. Prostate MR imaging: tissue characterization with pharmacokinetic volume and blood flow parameters and correlation with histologic parameters. Radiology. 2009;252:101-108.
42. Vos EK, Litjens GJ, Kobus T, et al. Assessment of prostate cancer aggressiveness using dynamic contrast-enhanced magnetic resonance imaging at $3 \mathrm{~T}$. Eur Urol. 2013;64:448-455.

43. Sakr WA, Tefilli MV, Grignon DJ, et al. Gleason score 7 prostate cancer: a heterogeneous entity? Correlation with pathologic parameters and diseasefree survival. Urology. 2000;56:730-734.

44. Goetti R, Leschka S, Desbiolles L, et al. Quantitative computed tomography liver perfusion imaging using dynamic spiral scanning with variable pitch feasibility and initial results in patients with cancer metastases. Invest Radiol. 2010;45:419-426.

45. Goh V, Dattani M, Farwell J, et al. Radiation dose from volumetric helical perfusion CT of the thorax, abdomen or pelvis. Eur Radiol. 2011;21:974-981. 